\title{
Role of Follicle-Stimulating Hormone and Androgens on the Sexual Inversion of Sevenband Grouper Epinephelus septemfasciatus
}

\author{
Richard J. Kline* and Izhar A. Khan \\ Marine Science Institute, University of Texas at Austin, Port Aransas, Texas 78373, USA \\ Kiyoshi Soyano and Megumi Takushima \\ Institute for East China Sea Research, Nagasaki University, Nagasaki 851-2213, Japan
}

\begin{abstract}
This study was designed to characterize the amount of androgen implant and the time period necessary to cause sexual inversion in sevenband grouper Epinephelus septemfasciatus and to determine whether administration of follicle-stimulating hormone (FSH) with or without androgens had any effect on the sexual state of this species. Seventy-two fish were treated with $10 \mu \mathrm{g}$ of recombinant FSH/kg body weight (BW) or saline control in cocoa butter. Two days later, cocoa butter and $\alpha$-cellulose pellet implants of control, $0.1 \mathrm{mg}$ of low-androgen mixture (LoAN)/kg BW, or $5 \mathrm{mg}$ of high-androgen mixture (HiAN)/kg BW were injected into the same fish. Fish were sampled after 3 and 5 weeks. Gonadosomatic indices (GSIs) at 3 weeks for FSH, HiAN, and FSH+HiAN treatment groups were significantly lower than the control values. At 5 weeks, no significant difference was observed, although the GSIs for HiAN treatment groups were lowest at both sampling points. The highest GSI values recorded were in the FSH-only treatment group at 5 weeks. All the controls remained in the immature female state throughout the study. High-dose androgen levels caused sexual transition of gonadal tissue at 3 weeks with spermatocytes present in all individuals. At 5 weeks, all individuals in the HiAN treatment group had complete inversion to male gonad, and sperm sinus development occurred in four individuals. However, incomplete sexual inversion was observed in the FSH $+\mathrm{HiAN}$ treatment groups with some ovarian tissue persisting even at 5 weeks. Exogenous FSH treatment alone appears to stimulate ovarian growth in the postspawning period. The 3-week period of sexual transition in this study is one of the shortest time periods recorded for grouper sexual inversion. This may be due to androgen treatment in the postspawning period, the proposed period of natural sex change in several grouper species.
\end{abstract}

The mahata or sevenband grouper Epinephelus septemfasciatus is a popular food fish species in the southwest region of Japan. Aquaculture of this species is of great interest owing to the high market value of wild-caught fish, which peaks in winter. In December 2006, the species peaked at 5,000 yen $/ \mathrm{kg}$ ( US\$42.62/ $\mathrm{kg}$ ), sold as whole fish (Nagasaki Fish Market, unpublished data). The sevenband grouper is a protogynous hermaphrodite that reaches female sexual maturity at age 4 and sexual inversion to male at age 7-8 (K. Soyano, unpublished). The spawning period for the sevenband grouper in Japan begins in the late spring and continues until early summer, and gonads regress in late summer (Shein et al. 2004). Obtaining male broodstock for captive culture is problematic owing to the advanced age at sexual inversion. Furthermore, additional information regarding the reproductive cycle and hormone action on gonads is needed for better reproductive management of this species in aquacul-

\footnotetext{
* Corresponding author: rkline@utmsi.utexas.edu
}

Received March 20, 2007; accepted July 18, 2007 Published online March 27, 2008 ture. Research on the maturation and spawning of this species is under way in Nagasaki, Japan, to develop techniques for sustainable aquaculture.

A variety of regulatory factors, such as sex steroids (Higa et al. 2003; Nakamura et al. 2003), enzyme pathway inhibitors (Bhandari et al. 2004; Kroon et al. 2005), and neurohormones (Watanabe et al. 1995; Kramer and Imbriano 1997), have been implicated in the sex change of hermaphroditic species. Owing to the capacity for sex change in many marine teleost species and the long time needed to change to the terminal sex, several studies have applied exogenous hormones to induce precocious sexual inversion in hermaphroditic fish (Hassin et al. 1997; Lee et al. 2000; Yeh et al. 2003a, 2003b). The most commonly used hormones are androgens, especially more potent synthetic forms, such as $17 \alpha$-methyltestosterone (MT; Yeh et al. 1989; Glamuzina et al. 1998; Mor et al. 2001). Methods of application vary, from addition to the feed (Kuo et al. 1988) to slow-release implants (Yeh et al. 1989). Results of individual studies in groupers vary in the amount of time necessary to induce complete sexual inversion, ranging from several months in the dusky 
grouper E. marginatus (Glamuzina et al. 1998) to 6 months in the blue-spotted grouper E. fario (Kuo et al. 1988). Previous studies on the orange-spotted grouper E. coioides and potato grouper E. tukula in Taiwan have shown that high-dose androgen implants containing a mixture of testosterone ( $\mathrm{T}$ ) and synthetic androgens at levels between 1 and $10 \mathrm{mg} / \mathrm{kg}$ body weight $(\mathrm{BW})$ were the most effective at inducing sex change over several months of treatment (Yeh et al. 2003a, 2003b). However, dosage and time requirements for hormone-induced sexual inversion by means of this method are variable in different species, and similar information is lacking for the sevenband grouper.

The gonadotropins (follicle-stimulating hormone $[\mathrm{FSH}]$ and luteinizing hormone [LH]) are important pituitary hormones that control development and maturation of gonads in teleost fishes (Miura et al. 1991; Swanson et al. 1991; Khan and Thomas 1999). Luteinizing hormone has been demonstrated to regulate all stages of gametogenesis in both male and female fish and has been shown to bind both $\mathrm{LH}$ and FSH receptors in the gonads of coho salmon Oncorhynchus kisutch and African catfish Clarias gariepinus (Miwa et al. 1994; Vischer et al. 2003). On the other hand, FSH has been shown to perform a more limited role in the initial oogenesis and spermatogenesis in gonochoristic models, such as salmon and catfish (Swanson et al. 1991; Vischer et al. 2003). The roles of these two gonadotropins are similar between the sexes in these two species of fish (Planas and Swanson 1995). However, these data are limited to a few families of fish, and data for other families (e.g., sea basses and groupers [family Serranidae] and porgies [family Sparidae]) suggest that a differential role of gonadotropins between the sexes may exist.

In the red seabream Pagrus major and black porgy Acanthopagrus schlegeli, some evidence of differential expression of FSH has been shown between the sexes. In the red seabream, a protandrous hermaphrodite, FSH messenger RNA (mRNA) levels in males drastically increase during the spawning season, while FSH mRNA levels in females remain low throughout all phases of the reproductive cycle (Gen et al. 2003). In the black porgy, which is a protogynous hermaphrodite, higher abundance of FSH receptor transcripts has been observed in testicular tissue than in ovarian tissue (Du et al. 2005), thereby suggesting a differential role for FSH in sexual inversion of hermaphroditic fishes. On the other hand, in the orange-spotted grouper, MT treatment in the feed caused a significant reduction in FSH mRNA transcript levels after $20 \mathrm{~d}$, while sexual inversion was occurring (Zhang et al. 2007). Clearly, more information on the role of FSH in relation to sex change is needed.

The present study was designed to (1) characterize the amount of androgen implant and the time period necessary to cause sexual inversion in sevenband grouper and (2) to determine whether administration of FSH with or without androgens had any effect on the sexual state of sevenband grouper.

\section{Methods}

Experimental animals.-Seventy-eight 5-year-old female sevenband grouper (BW, $1.16 \pm 0.02 \mathrm{~kg}$; total length, $44.0 \pm 0.2 \mathrm{~cm}$ [mean $\pm \mathrm{SE}]$ ), produced at the Nagasaki Prefectural Institute of Fisheries (Nagasaki, Japan) and housed in a large floating net pen, $\left(5 \mathrm{~m}^{3}\right)$ were used in this study. Six representative fish were sampled before the experiment to verify gonadal stage and approximate weight for treatment dosage. The remaining 72 fish were housed in two smaller net pens $\left(4 \mathrm{~m}^{3}\right)$ during the experiment. The fish were subjected to ambient photoperiod and temperature in the new Nagasaki Port area and fed a mixture of chopped fish and squid to satiation three times each week. Water temperature was recorded daily at the Nagasaki Institute of Fisheries with a digital thermometer. During all phases of the experiment, fish were evenly distributed and sampled from the two net pens.

Hormones and chemicals.-Androgens (T, MT, and testosterone propionate [TP]), cocoa butter, and $\alpha$-cellulose were purchased from Sigma Chemical Co. (St. Louis, Missouri). Recombinant FSH from red seabream raised in a baculovirus silkworm expression system was a gift from Koichiro Gen (National Research Institute of Fisheries, Japan).

FSH administration.-Initially, all fish were captured, anesthetized with 2-phenoxyethanol (0.05\%), and weighed to the nearest $10 \mathrm{~g}$. All fish were injected with a transponder tag for subsequent identification. Two groups of 36 fish each were injected with either saline and cocoa butter (control) or $10 \mu \mathrm{g}$ of FSH and cocoa butter/kg BW (treatment). Selection of this dose was based on the level of maximum effectiveness for red seabream (K. Gen, National Research Institute of Fisheries, unpublished data).

Androgen treatments.-After $2 \mathrm{~d}$, an androgen treatment was administered to each fish (Table 1). All fish were recaptured, anesthetized with 2-phenoxyethanol $(0.05 \%)$, identified via transponder tag, and implanted with an $\alpha$-cellulose-cocoa butter implant containing either $0,0.1 \mathrm{mg}$ low-androgen mixture $(\mathrm{LoAN}) / \mathrm{kg} \mathrm{BW}$, or $5 \mathrm{mg}$ high-androgen mixture (HiAN)/kg BW of T, MT, and TP at an equal weight ratio following the pellet design of Yeh et al. (1989). These pellets were implanted into each fish intramus- 
TABLE 1.-Experimental protocol (treatment and sampling regime) for the administration of follicle-stimulating hormone (FSH) and androgen at 3- and 5-week sampling periods in sexual inversion study conducted in seven band grouper. Treatments are as follows: FSH $=10 \mathrm{mg}$ recombinant red seabream FSH/kg body weight (BW), LoAN (low-androgen mixture) $=0.1 \mathrm{mg}$ androgen $/ \mathrm{kg} \mathrm{BW}$, HiAN (high-androgen mixture $)=5 \mathrm{mg}$ androgen $/ \mathrm{kg} \mathrm{BW}$.

\begin{tabular}{lcc}
\hline & \multicolumn{2}{c}{ Number of fish } \\
\cline { 2 - 3 } Treatment group & 3 weeks & 5 weeks \\
\hline Control & 6 & 6 \\
FSH & 6 & 6 \\
LoAN & 6 & 6 \\
HiAN & 6 & 6 \\
FSH + LoAN & 6 & 6 \\
FSH + HiAN & 6 & 6 \\
Total & 36 & 36 \\
\hline
\end{tabular}

cularly, behind the dorsal fin, and were prepared from the mixture of androgens with cocoa butter and $\alpha$ cellulose 3:6 weight ratio following the methods of Yeh et al. (1989). The control and treatment groups were evenly distributed between two net pens.

Sampling procedures.-Fish were sampled equally from the two net pens at 3 weeks (August 2) and 5 weeks (August 15) in the early morning, as shown in Table 1. Between treatment and the first sampling period, one fish died in the LoAN treatment group. All fish were anesthetized with 2-phenoxyethanol (0.05\%) and identified via transponder tag. All fish, gonad, and liver weights were recorded to the nearest gram. Hepatosomatic index (HSI) and gonadosomatic index (GSI) were calculated with the following formula:

$$
\left(\frac{\text { Organ weight }}{\text { Body weight }- \text { organ weight }}\right) \times 100 \text {. }
$$

For histological examination, gonadal tissue was sampled at several locations along the gonad and fixed in Bouin's solution for $24 \mathrm{~h}$. The samples were serially dehydrated and embedded in paraffin, and 3-5- $\mu \mathrm{m}$ transverse sections were made of each fixed tissue and stained with Mayer's hematoxylin and eosin. Each slide was examined with a light microscope and scored qualitatively for gonadal sex and stage similar to those in Bhandari et al. (2003) and Yeh et al. (2003a). Representative sections were digitized with a CoolSNAP-Pro color camera (Media Cybernetics, Silver Spring, Maryland) and QCapture Pro 5.0 software (QImaging, Burnaby, British Columbia).

Statistical analyses.-Data for HSI and GSI for did not meet the assumptions of normality for parametric tests, even when transformations were applied (Zar 1999). Therefore, differences in both GSI and HSI treatment groups were compared with Kruskal-Wallis one-way analysis of variance (ANOVA) followed by a post hoc Mann-Whitney $U$-test (Siegel and Castellan 1988). Differences between 3- and 5-week control groups were compared with the Mann-Whitney $U$-test. Significance was determined at $P<0.05$. All data are expressed as mean $\pm \mathrm{SE}$.

\section{Results}

Between the two sampling periods, the water temperature surrounding the floating net pens increased. Between the start of the study and week 3 , the temperature remained relatively constant at $28^{\circ} \mathrm{C}$. Between weeks 3 and 5 of the sampling periods, the temperature increased to more than $30^{\circ} \mathrm{C}$. Spawning temperature of this species is temperature dependent and ranges from $20^{\circ} \mathrm{C}$ to $25^{\circ} \mathrm{C}$ (Shein et al. 2004).

\section{Hepatosomatic Index}

There were no significant differences in HSI between the control and treatment groups at each sampling period. However, HSIs were significantly different in the controls between the two sampling periods. The mean HSI was $0.62 \pm 0.03 \%$ and $0.99 \pm$ $0.04 \%$ for the 3 - and 5-week sampling periods, respectively.

\section{Gonadosomatic Index}

There was no significant difference in the GSIs of fish between the initial control and 3-week control groups (data not included). The GSIs for the five treatments and controls during the 3- and 5-week sampling periods are displayed in Figure 1. At 3 weeks, the GSIs for the FSH, HiAN, and FSH+HiAN groups were significantly lower than those of the control group. No significant difference was observed between treatment groups; however, the lowest GSI was observed in the HiAN treatment groups (Figure 1, left panel).

At 5 weeks, the GSIs of control fish were significantly lower than for the 3-week sampling period. None of the 5-week treatment groups were significantly different from the corresponding control group. However, the lowest GSI was observed in the two HiAN treatment groups (Figure 1, right panel). The GSIs of the FSH treatment group were variable for both sampling periods, although the highest overall GSI values $(0.72,0.33$, and $0.27 \%)$ were observed in three fish at the 5-week sampling period (Figure 1, left panel).

\section{Histological Examination}

There was no difference in the gonadal histology of the initial control and 3-week control groups (data not included). The 3- and 5-week control groups showed 
3 Weeks

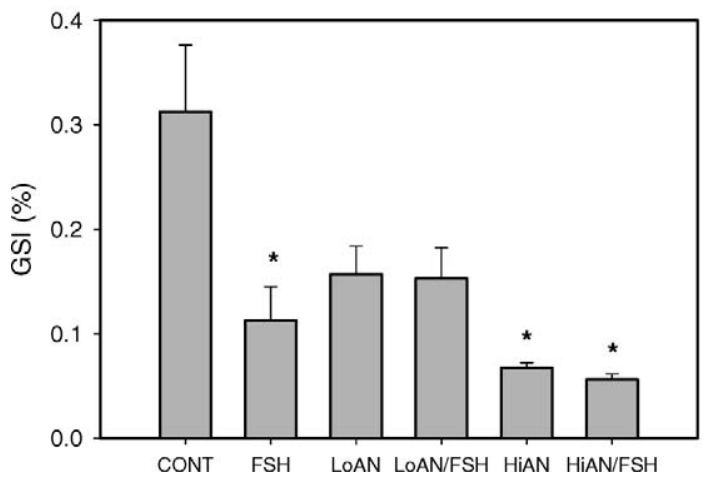

5 Weeks

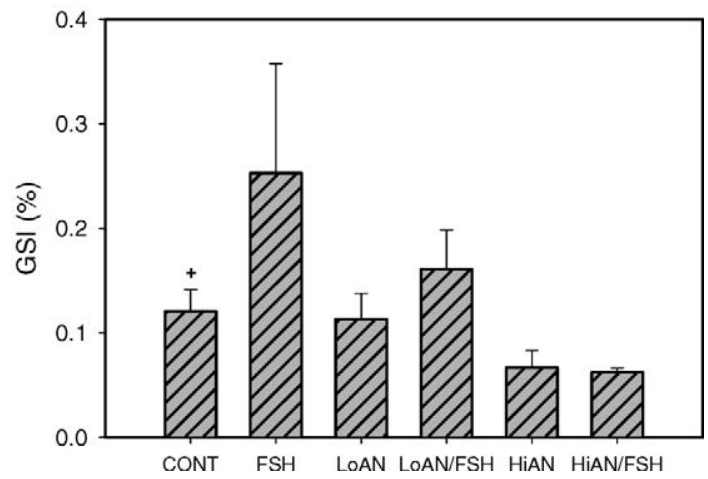

FIGURE 1.-Gonadosomatic indices (GSIs) of sevenband grouper at 3- and 5-week sampling periods. The asterisks indicate significantly lower than control, and the plus signs indicate significantly lower than 3-week control. Treatments are as follows: $\mathrm{FSH}=10 \mu \mathrm{g}$ recombinant red seabream $\mathrm{FSH} / \mathrm{kg}$ body weight $(\mathrm{BW}), \mathrm{LoAN}=0.1 \mathrm{mg}$ androgen $/ \mathrm{kg} \mathrm{BW}, \mathrm{HiAN}=5 \mathrm{mg}$ androgen/ $\mathrm{kg} \mathrm{BW}$. Each bar represents the mean $\pm \mathrm{SE}$ of six fish, except for the 3-week LoAN group where $n=5$. Abbreviations: CONT $=$ control, FSH = follicle-stimulating hormone, LoAN = low-androgen mixture, HiAN = high-androgen mixture.

no evidence of change from the F1 regressing female state. Ovaries consisted of gonia and perinucleolar stage oocytes (Figure 2A, G). The 5-week controls had much more degraded ovaries showing considerable atresia and phagocytosis along with more gonial cell proliferation (Figure 2G).

The tissue sections from the FSH treatment groups at 3 and 5 weeks contained all ovarian tissue (Figure 2B, $\mathrm{H})$ however this treatment group showed considerable changes not seen in the controls. At 3 weeks, gonial cell abundance had drastically increased in all fish and had proliferated throughout the ovarian lamellae (Figure 2B). Connective tissue and stromal tissue had also proliferated throughout the ovary. At 5 weeks, increased stromal and gonial cell proliferation was still evident in some samples. In three of six fish (Figure $2 \mathrm{H}$ ), oocytes were much larger and more developed, some advancing to the early cortical alveoli stage. Little atresia was present in the surrounding oocytes. In comparison to all other experimental groups, these three fish had much larger oocytes and well- formed ovarian lamellae.

In the LoAN treatment groups, the majority of the gonad tissue was female with a combination of perinucleolar and atretic oocytes along with gonial cells. At both 3 and 5 weeks, the LoAN treatment groups exhibited numerous small perinucleolar oocytes not seen in the controls (Figure 2C, I, respectively). Occasional spermatogenic tissue was also present in the form of spermatocytes (Figure 2C).

Gonad tissue from the FSH+LoAN treatment displayed some similarities with the FSH-only treatment. At 3 weeks, stromal tissue and vascularization were evident (Figure 2D), and at 5 weeks (Figure 2J), two fish had oocytes that were larger and appeared similar to those seen in the FSH-only treatment. However, the abundance of these cells was much reduced in the FSH+LoAN treatment when compared to the FSH-only treatments. Occasional spermatogenic tissue was seen in the form of spermatocytes. The gonadal structure in the 3- and 5-week FSH+LoAN treatment groups was distinctly ovarian, displaying a mixture of atretic and intact perinucleolar oocytes. In comparison to the controls and LoAN treatment groups, there was an increase in gonial cell abundance.

The gonadal tissue of fish treated with the HiAN dose had substantially changed at both sampling periods. In all fish from both sampling periods, the majority of the tissue was spermatogenic. At 3 weeks, the majority of the tissue was early stage (M1) testicular tissue demonstrating an abundance of spermatocytes and spermatids (Figure 2E). Any remaining oocytes were atretic, and yellow-brown bodies were present. At 5 weeks, very few atretic oocytes were present, a complete reconfiguration of the gonad occurring. The majority of the cells observed were at the late stages of spermatogenesis and had a preponderance of spermatids and spermatozoa (Figure 2L). In four of six individuals, distinctive sperm sinuses had formed indicating complete sexual transition to male (M2). Ovarian lamellae structure was absent from these samples.

Similar effects of reconfiguring the ovary to testis were observed in the FSH+HiAN and HiAN treatments. However, in the FSH+HiAN treatment, more ovarian tissue persisted along the periphery of the lamellae structure at 3 weeks (Figure 2F), and the ovarian lamellae structure persisted at 5 weeks (Figure 
3 weeks:

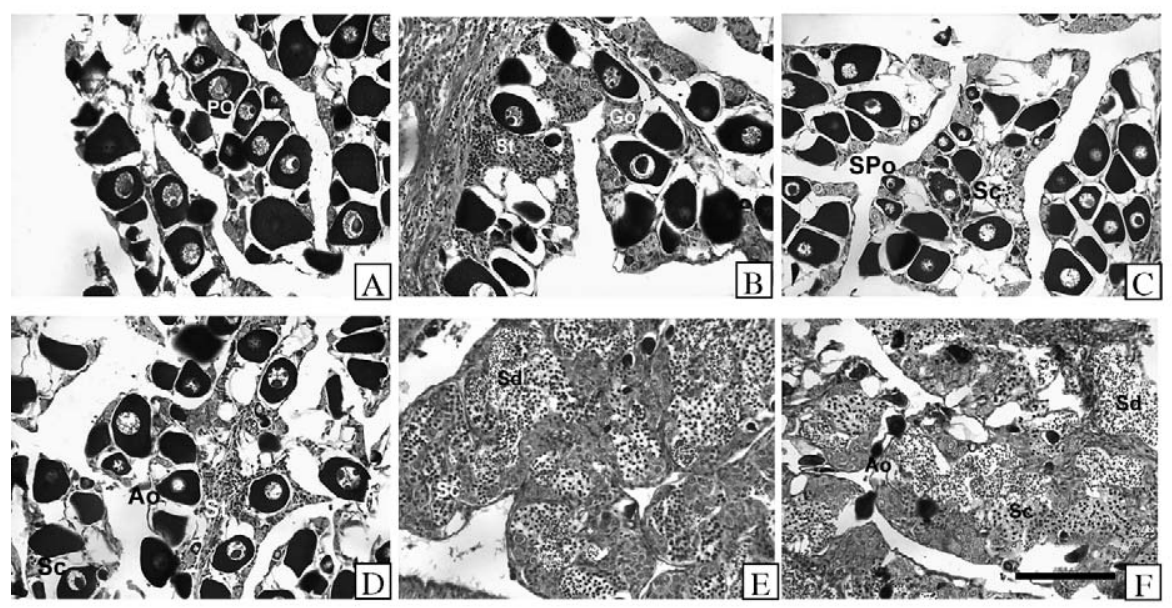

5 weeks:

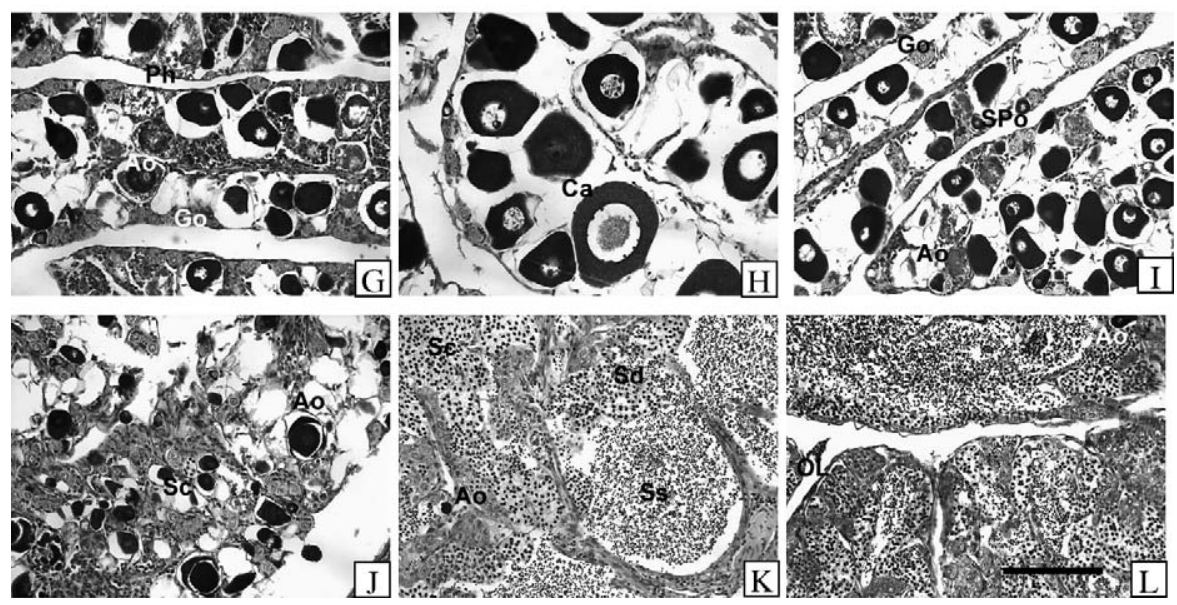

FIGURE 2.- Representative sections of gonadal tissue from sevenband grouper at 3- and 5-week sampling periods showing (A) control at 3 weeks, (B) follicle-stimulating hormone (FSH) treatment group at 3 weeks, (C) low-androgen (LoAN) treatment group: 0 at 3 weeks, (D) FSH+LoAN treatment group at 3 weeks, (E) high-androgen (HiAN) treatment group at 3 weeks, (F) FSH+HiAN treatment group at 3 weeks, (G) control at 5 weeks, (H) FSH treatment group at 5 weeks, (I) LoAN treatment group at 5 weeks, (J) FSH+LoAN treatment group at 5 weeks, (K) HiAN treatment group at 5 weeks, $(\mathbf{L})$ FSH+HiAN treatment group at 5 weeks. Treatments are as follows: $\mathrm{FSH}=10 \mu \mathrm{g}$ recombinant red seabream FSH/kg body weight $(\mathrm{BW}), \mathrm{LoAN}=0.1 \mathrm{mg}$ androgen $/ \mathrm{kg} \mathrm{BW}, \mathrm{HiAN}=5 \mathrm{mg}$ androgen $/ \mathrm{kg} \mathrm{BW}$. Abbreviations: $\mathrm{PO}=$ perinucleolar stage oocyte, $\mathrm{St}=$ stromal cells, $\mathrm{Go}=$ gonial cell, $\mathrm{SPo}=$ small new-growth perinucleolar oocyte, $\mathrm{Sc}=$ spermatocyte, Ao = atretic oocyte, $\mathrm{Sd}=\mathrm{spermatid}, \mathrm{Ph}=$ phagocytosis of oocyte, $\mathrm{Ca}=$ early cortical alveoli, $\mathrm{Ss}=$ sperm sinus with spermatozoa, $\mathrm{OL}=$ ovarian lamellae. Bar indicates $100 \mu \mathrm{m}$.

2L) where it had not in the HiAN treatment alone. Furthermore, the stage of development of the spermatogenic tissue was not as advanced as in $\mathrm{FSH}+\mathrm{HiAN}$ treatment alone. At 3 weeks, more gonial cells and spermatocytes were present along with many more oocytes around the periphery in comparison to HiAN treatment. At 5 weeks, more gonial cells and fewer spermatozoa were present in FSH+HiAN treatment compared to HiAN treatment alone. Howev- er, in two of six fish in the 5-week FSH+HiAN treatment group, sperm sinus had developed.

\section{Discussion}

This study demonstrates sexual inversion in sevenband grouper to the male M1 stage within 3 weeks of a high-androgen treatment with or without FSH pretreatment. By 5 weeks, most cells reach an advanced stage of spermatogenesis with a complete structural reorga- 
nization of the gonad. Similar results have been observed in other species of grouper using this technique (Yeh et al. 1989, 2003a, 2003b). These studies have examined only a small tissue sample collected with a cannula, which cannot provide detailed information regarding changes occurring throughout the gonad. The present study describes more detailed histological examination of the gonadal tissue after FSH and androgen treatments.

The $\alpha$-cellulose androgen mixture is a very potent technique to cause sexual inversion in hermaphroditic species, and fish have been successfully spawned by means of this technique as early as $90 \mathrm{~d}$ (Yeh et al. 2003a). In this study, histological observation of gonadal tissue revealed that treatment groups could be easily distinguished from the control groups by the stage of gonadal growth as early as 3 weeks after the treatments. The 3-week time period for complete sexual transition to the M1 stage for fish receiving high-androgen treatment is the one of the shortest time periods recorded in published studies for induced sexual inversion in groupers (Hassin et al. 1997; Glamuzina et al. 1998; Yeh et al. 2003b). This may be due to the timing of androgen treatment relative to the natural reproductive cycle in this species, which in the present study was the postspawning period. This is also the period for natural sexual inversion observed in several grouper species (Sadovy and Shapiro 1987; Bhandari et al. 2003) and, therefore, the fish are probably more receptive to androgen treatment at this stage.

The low dose of androgen $(0.1 \mathrm{mg} / \mathrm{kg})$ that caused sexual transition in two of seven orange-spotted grouper (Yeh et al. 2003b) was ineffective in causing sexual transition in sevenband grouper. However, the high-dose androgens caused sexual inversion in both species. The only apparent effect of the $0.1 \mathrm{mg} / \mathrm{kg}$ androgen implant in sevenband grouper was an increase in the abundance of small perinucleolar stage oocytes, which is consistent with the effects of lower androgen doses $(0.001$ and $0.01 \mathrm{mg} / \mathrm{kg})$ reported in orange-spotted grouper. It is unclear at this stage whether this effect of low-dose androgens is due to possible conversion of testosterone to estradiol or a direct action of androgens themselves.

Interestingly, FSH treatment alone at 3 weeks appears to stimulate reorganization of the gonad and growth of structural components such as stromal and vascular cells in sevenband grouper. At 5 weeks, the higher GSI values and increased oocyte diameters observed in several fish could be the result of additional cell growth (stromal tissue and gonia). This finding appears to be consistent with the data for orange-spotted grouper and red seabream, where MT treatment in the former species and 11-ketotestosterone (11-KT) treatment in the latter species down regulate FSH $\beta$ mRNA expression. It is possible that natural sexual inversion to the male state in sevenband grouper may require a down regulation of FSH by 11-KT production. In this study, exogenous FSH treatment appeared to slow the process of sexual inversion to the male state that was observed with high-androgen treatment alone. The persistence of lamellae structures and oocytes could indicate a prevention of apoptosis in the oocytes in the periphery of the ovarian lamellae. The FSH treatment before androgen implant could have initially driven growth of the gonad in the feminine direction that was then reversed by the subsequent androgen treatment. In other words, FSH may direct the gonad to grow initially, and the fate of the cells (gonial and stromal) is determined by the surrounding steroid environment. To investigate this, future studies could incorporate treatment with androgens followed by FSH to determine whether FSH plays a differential role in gonadal development between the sexes.

The increase in water temperature between sampling periods caused increased degradation of the gonad in the control fish. However, the FSH-treated fish and a few of the fish receiving FSH low-androgen treatment had much larger perinucleolar oocytes. Furthermore, both high-androgen treatments appeared more advanced at 5 weeks. This apparent temperature independence is intriguing and should be further investigated.

In conclusion, androgen implantation is a powerful tool for use in captive culture of sevenband grouper. This technique appears to work while the gonad is regressing in the postspawning season. Follicle-stimulating hormone appears to stimulate new cell growth in the postspawning period and to direct growth in the ovarian direction, inhibiting to some degree the effects of androgen alone.

\section{Acknowledgments}

We thank the members of the Soyano laboratory at the Center for East China Sea Research, Nagasaki University, for help with the experiments and processing of samples. The recombinant seabream FSH was provided by Koichiro Gen. This research was funded by the East Asia and Pacific Summer Studies Program and the Sid Richardson Memorial Fund. This is contribution 1194 of the University of Texas at Austin Marine Science Institute.

\section{References}

Bhandari, R. K., M. Higa, S. Nakamura, and M. Nakamura. 2004. Aromatase inhibitor induces complete sex change 
in the protogynous honeycomb grouper (Epinephelus merra). Molecular Reproduction and Development 67:303-307.

Bhandari, R. K., H. Komuro, S. Nakamura, M. Higa, and M. Nakamura. 2003. Gonadal restructuring and correlative steroid hormone profiles during natural sex change in protogynous honeycomb grouper (Epinephelus merra). Zoological Science 20:1399-1404.

Du, J. L., Y. H. Lee, W. S. Yueh, and C. F. Chang. 2005. Seasonal profiles of brain and pituitary gonadotropinreleasing hormone and plasma luteinizing hormone in relation to sex change of protandrous black porgy, Acanthopagrus schlegeli. Biology of Reproduction 72:922-931.

Gen, K., S. Yamaguchi, K. Okuzawa, N. Kumakura, H. Tanaka, and H. Kagawa. 2003. Physiological roles of FSH and LH in red seabream, Pagrus major. Fish Physiology and Biochemistry 28:77-80.

Glamuzina, B., N. Glavic, B. Skaramuca, and V. Kozul. 1998. Induced sex reversal of dusky grouper, Epinephelus marginatus (Lowe). Aquaculture Research 29:563-567.

Hassin, S., D. deMonbrison, Y. Hanin, A. Elizur, Y. Zohar, and D. M. Popper. 1997. Domestication of the white grouper, Epinephelus aeneus. 1. Growth and reproduction. Aquaculture 156:305-316.

Higa, M., K. Ogasawara, A. Sakaguchi, Y. Nagahama, and M. Nakamura. 2003. Role of steroid hormones in sex change of protogynous wrasse. Fish Physiology and Biochemistry $28: 149-150$.

Khan, I. A., and P. Thomas. 1999. Ovarian cycle, teleost fish. Pages 552-564 in E. Knobil and J. D. Neill, editors. Encyclopedia of reproduction, volume 3. Academic Press, San Diego, California.

Kramer, C. R., and M. A. Imbriano. 1997. Neuropeptide Y (NPY) induces gonad reversal in the protogynous bluehead wrasse, Thalassoma bifasciatum (Teleostei: Labridae). Journal of Experimental Zoology 279:133144.

Kroon, F. J., P. L. Munday, D. A. Westcott, J. P. A. Hobbs, and N. R. Liley. 2005. Aromatase pathway mediates sex change in each direction. Proceedings of the Royal Society Biological Sciences Series B 272:1399-1405.

Kuo, C. M., Y. Y. Ting, and S. L. Yeh. 1988. Induced sex reversal and spawning of blue-spotted grouper, Epinephelus fario. Aquaculture 74:113-126.

Lee, Y. H., F. Y. Lee, W. S. Yueh, P. Tacon, J. L. Du, C. N. Chang, S. R. Jeng, H. Tanaka, and C. F. Chang. 2000. Profiles of gonadal development, sex steroids, aromatase activity, and gonadotropin II in the controlled sex change of protandrous black porgy, Acanthopagrus schlegeli Bleeker. General and Comparative Endocrinology 119:111-120.

Miura, T., K. Yamauchi, H. Takahashi, and Y. Nagahama. 1991. Hormonal induction of all stages of spermatogenesis in vitro in the male Japanese eel (Anguilla japonica). Proceedings of the National Academy of Sciences of the USA 88:5774-5778.

Miwa, S., L. G. Yan, and P. Swanson. 1994. Localization of two gonadotropin receptors in the salmon gonad by in vitro ligand autoradiography. Biology of Reproduction 50:629-642.
Mor, G., M. Eliza, J. Song, B. Wiita, S. Chen, and F. Naftolin. 2001. 17 $\alpha$-Methyl testosterone is a competitive inhibitor of aromatase activity in Jar choriocarcinoma cells and macrophage-like THP-1 cells in culture. Journal of Steroid Biochemistry and Molecular Biology 79: 239-246.

Nakamura, M., R. K. Bhandari, and M. Higa. 2003. The role estrogens play in sex differentiation and sex changes of fish. Fish Physiology and Biochemistry 28:113-117.

Planas, J. V., and P. Swanson. 1995. Maturation-associated changes in the response of the salmon testis to the steroidogenic actions of gonadotropins (GTH-I and GTH-II) in vitro. Biology of Reproduction 52:697-704.

Sadovy, Y., and D. Y. Shapiro. 1987. Criteria for the diagnosis of hermaphroditism in fishes. Copeia 1987:136-156.

Shein, N. L., H. Chuda, T. Arakawa, K. Mizuno, and K. Soyano. 2004. Ovarian development and final oocyte maturation in cultured sevenband grouper Epinephelus septemfasciatus. Fisheries Science 70:360-365.

Siegel, S., and N. J. Castellan. 1988. Nonparametric statistics for the behavioral sciences, 2nd edition. McGraw-Hill, New York.

Swanson, P., K. Suzuki, H. Kawauchi, and W. W. Dickhoff. 1991. Isolation and characterization of two coho salmon gonadotropins, GTH-I and GTH-II. Biology of Reproduction 44:29-38.

Vischer, H. F., J. C. M. Granneman, M. H. K. Linskens, R. W. Schulz, and J. Bogerd. 2003. Both recombinant African catfish LH and FSH are able to activate the African catfish FSH receptor. Journal of Molecular Endocrinology 31:133-140.

Watanabe, W. O., S. C. Ellis, E. P. Ellis, W. D. Head, C. D. Kelley, A. Moriwake, C. S. Lee, and P. K. Bienfang. 1995. Progress in controlled breeding of Nassau grouper (Epinephelus striatus) broodstock by hormone induction. Aquaculture 138:205-219.

Yeh, S.-L., C.-M. Kuo, Y.-Y. Ting, and C.-F. Chang. 2003a. Androgens stimulate sex change in protogynous grouper, Epinephelus coioides: spawning performance in sexchanged males. Comparative Biochemistry and Physiology 135C:375-382.

Yeh, S.-L., C.-M. Kuo, Y.-Y. Ting, and C.-F. Chang. 2003b. The effects of exogenous androgens on ovarian development and sex change in female orange-spotted protogynous grouper, Epinephelus coioides. Aquaculture 218:729-739.

Yeh, S.-L., Y. Y. Ting, and C. M. Kuo. 1989. Technique of pellet implantation and preparation for induced sex reversal of the groupers Epinephelus salmonoides, Epinephelus fario. Bulletin of the Taiwan Fisheries Research Institute 47:213-219.

Zar, J. H. 1999. Biostatistical analysis, 4th edition. PrenticeHall, Upper Saddle River, New Jersey.

Zhang, W. M., Y. Zhang, L. H. Zhang, H. H. Zhao, X. Li, H. Huang, and H. R. Lin. 2007. The mRNA expression of $\mathrm{P} 450$ aromatase, gonadotropin $\beta$-subunits and FTZ-F1 in the orange-spotted grouper (Epinephelus coioides) during $17 \alpha$-methyltestosterone-induced precocious sex change. Molecular Reproduction and Development 74:665-673. 